\title{
Improving prefrontal cortex function in schizophrenia through focused training of cognitive control
}

\author{
Bethany G. Edwards', Deanna M. Barch ${ }^{1,2,3}$ and Todd S. Braver ${ }^{1,2 *}$ \\ 1 Departments of Psychology, Washington University, St. Louis, MO, USA \\ 2 Departments of Radiology, Washington University, St. Louis, MO, USA \\ ${ }^{3}$ Departments of Psychiatry, Washington University, St. Louis, MO, USA
}

\section{Edited by:}

Donald T. Stuss,

Baycrest Centre for Geriatric Care,

Canada; University of Toronto, Canada

\section{Reviewed by:}

Steven Silverstein,

University of Medicine and Dentistry of

New Jersey, USA

Cameron S. Carter,

University of California at Davis, USA

${ }^{*}$ Correspondence:

Todd S. Braver,

Department of Psychology, Campus

Box 1125, Washington University, St.

Louis, MO 63130, USA.

e-mail: tbraver@artsci.wustl.edu
Previous research has shown that individuals with schizophrenia show deficits in cognitive control functions thought to depend on the lateral prefrontal cortex (PFC), and its interactions with related regions. The current study explored the effects of instructed strategy training on improving cognitive control functioning in patients with schizophrenia. Event-related fMRI was used to test whether effects of such training were associated with changes in brain activity dynamics during task performance. Patients with schizophrenia $(N=22)$ performed the AXCPT cognitive control task in two-sessions, with the first occurring pre-training and second following strategy training. The training protocol emphasized direct encoding of contextual cues and updating response selection goals in accordance with cue information. A matched group of healthy controls $(N=14)$ underwent the same protocol but were only scanned in the pre-training session. In the pre-training session, patients exhibited behavioral evidence of impaired utilization of contextual cue information, along with reduced cue-related activity-but increased activation during probe and response periods - in a network of regions associated with cognitive control, centered on the lateral PFC. Following training, this pattern of activation dynamics significantly shifted, normalizing towards the pattern observed in controls. These activation effects were associated with both clinical symptoms and behavioral performance improvements. The results suggest that focused strategy training may facilitate cognitive task performance in patients with schizophrenia by changing the dynamics of activity within critical control-related brain regions.

Keywords: cognitive training, functional imaging, schizophrenia, cognitive control, prefrontal cortex, remediation

\section{INTRODUCTION}

The ability to guide task performance by priming goal-directed behavior and inhibiting habitual response tendencies is commonly referred to as cognitive control. It is reasonably well-established that patients with schizophrenia show significant disturbances in the ability to exert cognitive control (Cohen and Servan-Schreiber, 1992; Cohen et al., 1996; Braver et al., 1999; Reichenberg and Harvey, 2007). These impairments are reflected in both behavior and altered patterns of brain activity in the network of regions thought to support cognitive control, most prominently including the lateral PFC (Cohen and Servan-Schreiber, 1992; Braver et al., 1999; Cohen et al., 1999; MacDonald et al., 2000; Barch et al., 2001; MacDonald and Carter, 2003). In prior work, we have explored the hypothesis that patients with schizophrenia have a fundamental impairment in specific goalrelated functions mediated by the lateral PFC. In the current study, we investigate this hypothesis further, by examining whether explicit training in the use of contextual cue information in patients with schizophrenia is associated with normalizing effects on behavioral and brain activity markers of enhanced cognitive control, particularly those related to lateral PFC function.

A large body of accumulating evidence from brain imaging studies, as well as related work from other methodologies, has implicated the lateral PFC as a central component related to cognitive dysfunc- tion in schizophrenia (Goldman-Rakic, 1991; Goldman-Rakic and Selemon, 1997; Davidson and Heinrichs, 2003; Glahn et al., 2005; Van Snellenberg et al., 2006). In addition, a number of researchers have suggested that these lateral PFC deficits may be the result of - at least in part-dysregulated input from the midbrain dopamine (DA) system (Braver and Cohen, 2000; Braver et al., 1999). In our work, we have developed a theoretical framework variously termed context processing, goal maintenance, or guided activation, which suggests that goal-related information is represented and maintained in lateral PFC to guide, bias, and constrain on-going task processing (Cohen et al., 1999; O'Reilly et al., 1999; Barch et al., 2001; Braver et al., 2002). Further, we have argued that dysfunction in lateral PFC, and more specifically disrupted DA-PFC interactions, results in patients with schizophrenia having impairments in their ability to activate, update, and maintain goal-information based on contextual cues (Braver et al., 1999). More recently, we have refined this idea through the Dual Mechanisms of Control (DMC) account, which postulates two distinct modes of cognitive control: proactive and reactive (Braver et al., 2007, 2009). Proactive control depends upon the active maintenance of goal-related information in a sustained/anticipatory manner prior to the occurrence of cognitively demanding events. In contrast, reactive control is late-acting and is mobilized only as needed, such as in response to the detection of a high-interference event. The reactive form of 
control is less effective, since it relies upon quick, transient, and just-in-time forms of interference resolution. However, proactive control may be more vulnerable to disruption, since it is more resource demanding, and is postulated to be more dependent upon precise DA-PFC interactions (which enable appropriate updating and maintenance of goal representations). Thus, we have suggested that populations characterized by DA-PFC dysfunction (such as schizophrenia) may show a shift from proactive to reactive control, since the reactive control mode may be more robust in the face of such dysfunction.

In our investigations of schizophrenia, lateral PFC function, and more recently the DMC account, we have made frequent use of an AX version of the Continuous Performance Task (AX-CPT), since it provides a relatively specific probe of goal representation, maintenance, and updating (Servan-Schreiber et al., 1996; Barch et al., 1997; Cohen et al., 1999; Paxton et al., 2008). In this task, contextual cues serve as task goals regarding the appropriate actions to make in response to ambiguous probes. Active maintenance of this frequently updated task goal information is required to bridge the delay between cue and probe presentation. In a series of studies, it has been shown that the AX-CPT task provides sensitive and specific indices of cognitive control impairment in schizophrenia (Barch and Braver, 2005). Likewise, neuroimaging studies have reliably demonstrated that patients show alterations in lateral PFC activity related to the active maintenance of task-goals during AXCPT performance (Barch et al., 2001; Perlstein et al., 2003; Holmes et al., 2005; MacDonald et al., 2005; Yoon et al., 2008). Importantly, the DMC account makes specific predictions about the patterns of brain activity dynamics associated with proactive versus reactive control modes (Braver et al., 2009). Proactive control should be associated with increased anticipatory activation in lateral PFC, and emerge at the time of contextual cues in tasks such as the AX-CPT. In contrast, reactive control is predicted to be associated with reduced cue-related activity, but increased activation during probe periods (especially when probes have the potential to produce interference). Consistent with the hypothesis that individuals with schizophrenia show reduced proactive control and a shift to reactive control, previous neuroimaging studies of the AX-CPT have shown reduced cue-related activation in lateral PFC in patients with schizophrenia (e.g., MacDonald and Carter, 2003), although up to this point, no studies have reported analyses directly comparing cue and probe-related activation.

The goal of the current study is provide a more direct test of the DMC account in schizophrenia, by examining the dynamics of brain activity during cue and probe periods of the AX-CPT task trials. An additional goal is to show that the dynamics of brain activity might not be fixed in patients with schizophrenia, but could potentially be normalized towards the pattern exhibited by healthy controls. One possible route towards normalization could be through explicit cognitive training on the effective use of contextual information. Indeed, there is now a growing interest in cognitive training approaches in schizophrenia (Corrigan et al., 1995; Wykes et al., 2002a; Twamley et al., 2003, 2008; Fisher et al., 2009b; Haut et al., submitted), with some evidence of success at rehabilitation of impairments in different domains (Corrigan et al., 1995; Twamley et al., 2008; Fisher et al., 2009b; Haut et al., submitted). There have even been indications that such training approaches can alter brain function in regions such as lateral PFC (Wykes et al., 2002a), suggesting that there may be significant plasticity in the activation dynamics of these regions. Moreover, in our own prior work, we have demonstrated success with this approach in healthy older adults, who represent another population thought to suffer from (a milder form of) cognitive control impairment related to changes in DA-PFC interactions. Specifically, prior to cognitive training, older adults showed the reactive control pattern within lateral PFC and related regions (i.e., reduced cue-related but increased probe-related activation, Paxton et al., 2008), that we are also postulating to be present in patients with schizophrenia. However, following training, there was a significant shift in the activation dynamics in these same regions toward a more proactive pattern (i.e., cue-related activity increased while probe-related activity decreased, Braver et al., 2009). Thus, our goals for the current study were to demonstrate that: (1) prior to training, individuals with schizophrenia show lateral PFC dynamics consistent with a greater use of reactive control compared to healthy individuals; and (2) these same patients show a shift from reactive to proactive control following the identical training protocol previously employed in older adults.

\section{MATERIALS AND METHODS PARTICIPANTS}

Participants included 22 medicated patients with schizophrenia (4 females) and 14 healthy controls (3 females). An additional 14 participants ( 7 schizophrenia, 7 control) participated in the study, but were excluded from analysis due to excessive movement during scanning, poor signal-to-noise ratios, incomplete functional neuroimaging runs, or inability to accurately understand and perform the required task. The excluded controls did not differ on any demographic variable. Excluded patients had lower personal education $(t(26)=2.15, p=0.04)$, tended to be older $(t(26)=1.87, p=0.07)$, and had slightly higher disorganization symptoms $(t(26)=1.88$, $p=0.07)$. All participants were recruited through the Conte Center for the Neuroscience of Mental Disorders at Washington University. Trained research personnel collected diagnostic information through a Structural Clinical Interview for DSM-IV-TR (SCID-IV-TR, (First et al., 2001), which was then verified through medical record reviews. Out of the 22 patients, 16 were diagnosed with schizophrenia and 6 were diagnosed with schizoaffective disorder. Patients and controls were matched in regards to age, sex, ethnicity, and parental education (see Table 1). Participants (either schizophrenia or control) were excluded based on the following: non-English native language, meeting DSM-IV-TR criteria for substance abuse or dependence within the past 6 months, head injury with documented neurological sequelae or significant loss of consciousness, meeting DSM-IV-TR criteria for mental retardation (IQ below 70), or pregnancy or nursing. In addition, healthy controls were excluded for any history of an Axis I psychotic disorder, current diagnosis of major depression, first-degree family history of psychosis, or current treatment with psychotropic medications. All participants signed informed consent prior to participation in the study and all experimental procedures were approved by the Institutional Review Board (IRB) of Washington University and compiled with regulations. Participants received $\$ 25 /$ hour for their participation. 
Table 1 | Clinical and demographic characteristics.

\begin{tabular}{lll}
\hline Trial type & Patient mean (SD) & Control mean (SD) \\
\hline Age (years) & $34.8(9.7)$ & $36.3(8.1)$ \\
Sex (\% male) & 82 & 79 \\
Education (years) & $13.4(2.1)$ & $14.5(3.1)$ \\
Father Education (years) & $13.2(3.0)$ & $12.7(1.6)$ \\
Mother Education (years) & $13.2(3.5)$ & $12.9(2.3)$ \\
Handedness (\% right) & 91 & 100 \\
Mean SAPS Score & $3.5(2.4)$ & - \\
Mean SANS Score & $7.7(2.9)$ & - \\
Disorganization & $1.9(2.3)$ & - \\
\hline
\end{tabular}

SAPS, Structured Assessment of Positive Symptoms; SANS, Structured Assessment of Negative Symptoms.

\section{CLINICAL SYMPTOM RATINGS}

To assess symptom severity, patients were administered the Scales for the Assessment of both Positive and Negative Symptoms (Andreasen, 1983a,b) by a trained Master's level clinician. Consistent with prior schizophrenia research, we focused on scores from three primary symptom dimensions (Andreasen and Olsen, 1982; Lewine et al., 1983; Andreasen, 1985; Liddle, 1987; Miller et al., 1993; Toomey et al., 1997); positive, negative, and disorganized symptoms. The positive symptom dimension was comprised of the global scores for delusions and hallucinations while the negative symptom dimension was comprised of the global scores for alogia, affective flattening, avolition, and anhedonia. The disorganization symptom dimension was comprised of global scores for bizarre behavior, formal thought disorder, and attention. Patients also completed additional measures including the Wechsler Adult Intelligence Scale, Third Edition (WAIS-III; Wechsler, 1997) and the Edinburgh Handedness Scale (Oldfield, 1971).

\section{TASK}

Participants were asked to perform a version of the AX-CPT that taps into how contextual cues can be used to activate and update goal representations. In this AX-CPT task, participants are presented with cue-probe pairs (individual letters appearing on a computer screen in sequence). Target responses are to be made to the presentation of an X probe, but only when it follows an A cue. The majority of trials (70\%) are these AX targets. The remaining $30 \%$ of trials are split evenly between three types of non-target trials: (a) AY trials in which a valid cue $(A)$ is followed by an invalid probe (non $\mathrm{X}$, referred to as $\mathrm{Y}$ ), (b) BX trials in which an invalid cue (not A, referred to as $\mathrm{B}$ ) is followed by a valid probe (X), and (c) BY trials in which an invalid cue (not A) is followed by an invalid probe (not $\mathrm{X})$. The ability to use context is assessed by investigating patterns of performance on the two most challenging trial types, AY and BX. Because AX trials occur more often than any other trial type, participants are biased to make a target response when they see an $\mathrm{X}$ probe, even when A was not the cue letter (i.e., BX trials). In order to make a non-target response to these BX trials, contextual information provided by the cue letter must be used to inhibit the tendency to make a target response. Another bias occurs when the cue letter is an A and the probe letter is a letter other than X (i.e., AY trials). Again, because $70 \%$ of trials are AX trials, individuals with strong contextual representations develop an attentional expectancy for an $\mathrm{X}$ cue following an A and must overcome this invalid expectation in order to make a correct non-target response on AY trials. The proactive use of context information should lead to impaired AY performance but enhanced BX performance. Conversely, individuals with impaired proactive goal maintenance should show poorer performance on BX compared to AY trials.

Participants were instructed to respond by pressing one button to probes on target trials and a separate button to probes on non-target trials. Responses to cues were also made by pressing the non-target button. The total trial duration was $9100 \mathrm{~ms}$. The cue was presented on the screen for $300 \mathrm{~ms}$, followed by a 5000-ms delay prior to the probe. The probe was displayed for $300 \mathrm{~ms}$. Participants were given the full $1300 \mathrm{~ms}$ (probe and delay time) to respond to the probe by pressing either the target or non-target buttons. Each trial ended with a feedback message $(2500 \mathrm{~ms})$ indicating that the trial was over. A total of 120 trials were performed of which 84 were AX trials and 12 each were AY, BX, and BY trials.

\section{PROCEDURE}

Patients performed the AX-CPT task in two-sessions, with the first session occurring pre-training and the second immediately following strategy training. A matched group of healthy controls also performed the AX-CPT but were only scanned in the pre-training session. During the pre-training session, participants were given standard instructions for the task followed by 10-20 practice trials prior to going in the scanner. Participants then performed 3 blocks of 100 trials inside the scanner. The patients returned to the scanner for a second session on a separate day (separated by an average of 1 week) to receive proactive strategy training. The training protocol was identical to that used in Paxton et al. (2006) and Braver et al. (2009) with older adults on the AX-CPT and is described below. Immediately after completing the training, the patients performed the AX-CPT task again in the scanner with the identical protocol and procedure as employed in the first session.

\section{TRAINING PROTOCOL}

First participants were explicitly told that $70 \%$ of the trials in the task were an A cue followed by an X probe and would require the appropriate target response. They were also informed that the investigators were interested in whether explicitly discussing specific approaches to the task would change how people perform. Participants were instructed to first pay attention to the cue letter and decide whether it was an A or not. If the cue letter was an A, they were encouraged to prepare for an $\mathrm{X}$ and push the target button. If the cue letter was not an A, they were encouraged to prepare for a non-target button press regardless of what the probe letter was. They were trained to verbally categorize the cue letter by saying "A" or "not A" aloud while attending to the cue for 3 blocks of 10 trials each. For the first of the three blocks, the experimenter verbally categorized the cue letters, followed by the participant verbally categorizing the cue letters for the second block. Finally, for the third block, the participant verbally categorized the cue letters while completing the task. Participants were then trained to use the cue to influence how they would prepare for the probe. They were reminded that when the cue was an A it was very likely that an $\mathrm{X}$ would follow; therefore, they should begin to prepare for a target response. Participants were instructed to say 
"if X, target" when they saw an A as the cue and "non-target" when they saw a letter other than A as the cue for 6 blocks of 10 trials each. For the first block, the experimenter said these phrases aloud as the participant completed the task; the examiner and participant then switched roles for the second block. For the third block, the participant and examiner said these phrases aloud together and finally, for the remaining 3 blocks, the participant said these phrases aloud while completing the task. Training took approximately $20 \mathrm{~min}$ to complete. Following training, patients completed 3 task blocks of 100 trials in the scanner. They were reminded to use the strategy cues during each block, saying the strategy phrases only in their head and not aloud. Following the task blocks, participants were given debriefing questionnaires focusing on their perception and utilization of the training strategy.

\section{FUNCTIONAL IMAGING}

Structural and functional images were acquired on a head-only Siemens 3 Tesla Allegra System (Erlangen, Germany). A pillow and tape was used to minimize head movement in the head coil. Headphones dampened scanner noise and enabled communication with participants. Anatomical images were acquired using an MP-RAGE T1-weighted sequence. Functional images were acquired using an asymmetric spin-echo echo-planar sequence sensitive to blood-oxygen-level-dependent (BOLD) magnetic susceptibility $\left(\mathrm{TR}=2500 \mathrm{~ms} ; \mathrm{TE}=50 \mathrm{~ms} ;\right.$ Flip $\left.=90^{\circ}\right)$. Functional images were 40 contiguous slices of $3.75-\mathrm{mm}$ thickness acquired parallel to the anterior-posterior commissure line allowing complete brain coverage at a high signal-to-noise ratio (Conturo et al., 1996). Each run consisted of alternating blocks of task (2 per run) and fixation (3 per run). Task blocks were 60 trials in duration while fixation blocks (denoted by a centrally presented crosshair) were $25 \mathrm{~s}$ in duration. The first four images in each scanning run were used to allow the scanner to reach a steady state and were discarded. All functional images were corrected for movement (Friston et al., 1996; Snyder, 1996), and then registered to the participant's anatomical images. The data were then whole-brain normalized to a fixed value and spatially smoothed with a $9 \mathrm{~mm}$ full-width half-maximal Gaussian kernel. Participants' structural images were transformed into standardized atlas space (Talairach and Tournoux, 1988) using a 12-dimensional affine transformation (Woods et al., 1992, 1998). The functional images were then registered to the reference brain using the alignment parameters derived for the structural scans. Event-related activation was estimated with a general linear model (Friston et al., 1995). A delta-function approach was used in which each time-point of the event-related epoch was estimated separately, using a 25-s (10 TR) duration for the epoch. Parameter estimates from each participant's GLM were submitted to second-level tests treating participants as a random factor in $t$-tests and ANOVA.

Visual stimuli were presented using PsyScope software (Cohen et al., 1993) running on an Apple PowerMac G4. The letters were presented in white 48-point uppercase bold Helvetica font on a black screen. Stimuli were projected to participants with an LCD projector onto a screen positioned at the head end of the bore. Participants viewed the screen through a mirror attached to the head coil. A fiber-optic, light-sensitive key press interfaced with the PsyScope Button Box was used to record participants' behavioral performance.
We had specific hypotheses about how brain regions involved in the cognitive control network would behave both in terms of group differences in activation dynamics prior to training, and in terms of the effects of training on these dynamics. Thus, we used a very stringent approach to test for such effects. First, we used an ROI-based approach to identify cognitive control regions that showed the predicted group differences at baseline, and then used these regions to test focused hypotheses about training effects. To define the regions included in the cognitive control network, we created a mask of spherical ROIs (10-mm radius) using anatomical coordinates of regions described in two published meta-analyses as seed points (Wager and Smith, 2003; Owen et al., 2005). We have used this identical mask successfully in prior published studies (Emery et al., 2008; Fales et al., 2008). These ROI masks were used to constrain analysis to only those voxels that are theoretically most strongly associated with cognitive control. Second, we identified voxel clusters from within these masks that showed particular effects of interest. We started by identifying regions that showed the specific pattern of group differences predicted by the DMC theory, which was reduced cue-related activity-but increased probe related activity - in individuals with schizophrenia compared to controls. To identify this pattern we constructed multiple contrasts (a group $\mathrm{X}$ cue vs. probe interaction; $\mathrm{CON}>\mathrm{SCZ}$ for cue, $\mathrm{SCZ}>\mathrm{CON}$ for probe), and a voxel cluster was only identified if it simultaneously satisfied all of the contrasts $(p<0.05$, minimum cluster size $=8$ voxels). Third, we treated these identified clusters as whole ROIs to examine more detailed patterns of group differences in baseline activity. Fourth, we interrogated the activity in these identified group-difference regions to examine the effects of training on individuals with schizophrenia. Note that the investigation of training effects was ROI-based, and most importantly, was completely independent from the analysis that identified and defined the relevant ROIs. Thus, we believe that the analysis approach described below was sufficiently stringent in balancing concerns regarding both false positive and false negatives (as the latter is often overlooked, though it is just as important as false positives).

\section{RESULTS BEHAVIORAL \\ Group Differences}

To examine the use of proactive control more closely, we focused our analyses on AY and BX trial types, although data on all trial types are reported in Table 2. Error rate and RT data were both analyzed using an ANOVA with group (patient vs. control) as the between-subjects variable and trial type (AY vs. BX) as a withinsubject variable. The ANOVA for errors indicated a main effect of group, $F(1,34)=5.11, p<0.05$, due to greater errors in patients on both AY and BX trials. The RT ANOVA also indicated a main effect of group, $F(1,34)=4.96, p<0.05$, with patients responding significantly slower than healthy controls for $\mathrm{AY}$ and $\mathrm{BX}$ trials types (AY: $t(34)=2.04, p=0.05$; BX: $t(34)=2.03, p=0.05)$. A main effect of trial type was also observed in RT, $F(1,34)=24.03, p<0.001$. Both patients and controls responded slower on AY than BX trials (Patients: $t(21)=2.94, p<0.01$; Controls: $t(13)=5.37, p<0.001$ ). We also derived a more focused behavioral index of proactive control by combining performance across $\mathrm{AY}$ and $\mathrm{BX}$ trial types, using the formula $[(\mathrm{AY}-\mathrm{BX}) /(\mathrm{AY}+\mathrm{BX})]$ (Paxton et al., 2006, 2008). As 
Table 2 | Proportion of errors and reaction times.

\begin{tabular}{llll}
\hline TrialType & \multicolumn{2}{c}{$\begin{array}{c}\text { Pre-training } \\
\text { session }\end{array}$} & $\begin{array}{c}\text { Post-training } \\
\text { session }\end{array}$ \\
\cline { 2 - 3 } & $\begin{array}{l}\text { Patient } \\
\text { mean (SD) }\end{array}$ & $\begin{array}{l}\text { Control } \\
\text { mean (SD) }\end{array}$ & Patient \\
& & & \\
\hline ERRORS & & & \\
AX & $0.14(0.17)$ & $0.03(0.06)$ & $0.12(0.17)$ \\
AY & $0.19(0.19)$ & $0.09(0.10)$ & $0.24(0.23)$ \\
BX & $0.28(0.26)$ & $0.13(0.18)$ & $0.21(0.27)$ \\
BY & $0.07(0.16)$ & $0.04(0.08)$ & $0.08(0.13)$ \\
RT & & & $669(177)$ \\
AX & $716(145)$ & $596(143)$ & $852(141)$ \\
AY & $868(145)$ & $756(184)$ & $675(238)$ \\
BX & $749(228)$ & $601(191)$ & $648(195)$ \\
BY & $696(192)$ & $576(198)$ & \\
\hline
\end{tabular}

described in the methods, use of proactive control should lead to increased AY but decreased BX errors. Thus, negative scores on this behavioral measure indicate poor use of proactive control while positive scores indicate better use of proactive control. The index of proactive control was computed for error rate, reaction time, and the sum of error rate and reaction time (after $z$-scoring each measure to put them on the same scale). Planned contrasts showed that prior to training (see Table 3), healthy controls demonstrate significantly more proactive control in terms of both errors and RT than patients, but only the RT effect was statistically significant (RT: $F(1,34)=3.28, p<0.001$; error: $F(1,34)=0.04, p=0.25$; sum: $F(1,34)=1.43, p=0.85)$.

Although the index of proactive control described above was significantly reduced for patients compared to controls in RT, overall the pattern of group differences was not as strong as predicted. The most common pattern observed in studies of the AX-CPT in schizophrenia is a group $\times$ trial type interaction, caused by disproportionately poorer performance on $\mathrm{BX}$ than $\mathrm{AY}$ trials in patients (with some studies even showing improved performance on AY trials among patients (Javitt et al., 2000; Barch et al., 2001, 2003). However, in the current study, only simple group main effects were observed. The absence of a group $\times$ trial type interaction could be explained by a few factors. First, although patients still made many more BX than AY errors at baseline, AY errors were elevated in patients relative to what is typically found in this task. Second, controls also made more BX than AY errors, which is not the typical pattern for controls (i.e., usually more $\mathrm{AY}$ than BX errors) (Barch et al., 2001; MacDonald et al., 2003). Third, patients had slower performance on $\mathrm{AY}$ trials relative to $\mathrm{BX}$, an effect usually seen only in controls. It is important to note that although this pattern was somewhat different from prior studies, it does not indicate that either patients or controls were performing the task inappropriately. Indeed, errors and RT on the two trial types used as internal controls - AX and BY - were similar to what has been observed in prior studies, and indicate that both groups were performing the task at reasonably proficient levels. Moreover, the fact that the patient group showed a behavioral pattern somewhat similar to controls makes the imaging data all the more important, as it provides a
Table 3 | Context processing scores for Error, RT, and sum of Error and RT.

\begin{tabular}{lrrl}
\hline & \multicolumn{2}{c}{$\begin{array}{c}\text { Pre-training } \\
\text { session }\end{array}$} & $\begin{array}{l}\text { Post-training } \\
\text { session }\end{array}$ \\
\cline { 2 - 3 } & $\begin{array}{l}\text { Patient } \\
\text { mean (SD) }\end{array}$ & $\begin{array}{l}\text { Control } \\
\text { mean (SD) }\end{array}$ & $\begin{array}{l}\text { Patient } \\
\text { mean (SD) }\end{array}$ \\
\hline Error & $-0.24(0.56)$ & $-0.16(0.44)$ & $0.03(0.62)$ \\
RT & $0.09(0.14)$ & $0.12(0.09)$ & $0.14(0.12)$ \\
Sum & $-0.15(0.64)$ & $-0.04(0.47)$ & $0.17(0.67)$ \\
\hline
\end{tabular}

stronger test of whether the underlying dynamics of brain function differ between the two groups even when the endpoint behavior is qualitatively similar. Although such explanations are always posthoc, we believe that this somewhat atypical pattern of behavioral results might reflect the fact that this was a somewhat more functional sample of individuals with schizophrenia than we normally recruit. This is because these individuals needed to be able to scan twice in a relatively short period of time and had to be willing to engage in the training process. This hypothesis is supported by the relatively high average education scores of the patient sample (at least 1 year of college on average) and the fact that the symptom scores were relatively low.

\section{Training Effects}

To examine patient training effects, errors and RTs were analyzed in an ANOVA with session (pre-training vs. post-training) and trial type (AY vs. BX) as within-subject variables. The ANOVA for errors indicated a significant session $\times$ trial type interaction, $F(1$, $21)=6.06, p<0.05$, with BX errors significantly decreased posttraining relative to pre-training $(t(21)=2.08, p=0.05)$. For AY trials, the opposite, but predicted pattern was present, with a trainingrelated increase in errors, though the effect did not reach significance $(t(21)=-1.38, p=0.18)$. The RT ANOVA indicated a main effect of session, $F(1,21)=4.96, p<0.05$. Planned contrasts showed that patients responded significantly faster to BX trials following training $(t(21)=2.85, p=0.01)$, but, as predicted, did not speed up on AY trials $(t(21)=0.64, p=0.53)$. However, the differential effect of training on $\mathrm{AY}$ and $\mathrm{BX}$ was not strong enough to produce a session $\times$ trial type interaction $(F(1,21)=3.51, p>0.1)$. We further examined training effects on proactive control indices and found that patients showed significant improvement in proactive control following training in terms of reaction time $(t(21)=-2.24, p<0.05)$ and the sum of error rate and reaction time $(t(21)=-2.06, p=0.05)$. We then correlated behavioral performance with patient self-report answers to whether they were approaching the task in any particular way and found that patients who indicated that they were following our training strategy showed more training-related BX improvement in terms of RT (PST-PRE BX RT: $r=-0.53, p=0.01$ ).

\section{Relationship between task performance and symptoms}

Finally, we examined whether task performance was associated with patient symptoms. Prior to training, poorer performance on BX trials (more BX errors) was associated with higher disorganized symptoms $(r=0.51, p<0.05)$. Further, patients with more disorganized symptoms demonstrated a greater benefit of 
training in terms of a larger reduction in BX errors $(r=-0.55$, $p<0.01)$. Lastly, lower RT proactive control was associated with more disorganized symptoms (pre-training, $r=-0.43, p=0.05$; post-training, $r=-0.51, p<0.05)$, although disorganization was not associated with the magnitude of change in the proactive control indices.

\section{FUNCTIONAL IMAGING \\ Group Differences}

We first examined significant differences between patients and healthy controls prior to training, focusing our analyses on the event-related dynamics of brain activity in our a priori ROIs (see methods for details). Specifically, we hypothesized that impaired proactive control in patients would be reflected in reduced cuerelated and increased probe-related activation relative to controls. We tested this hypothesis by identifying regions within the cognitive control network showing a group $\times$ event type (cue vs. probe) interaction of a specific cross-over pattern (cue: patients < controls; probe: patients > controls). The cue event was defined as the average activation over time points 3 and 4 (5.0-7.5 s following the start of the trial and cue onset), while the probe event was defined as the average activation over time points 5 and 6 (10.0-12.5 s following start of trial, 5.0-7.5 s following probe onset - see Figure 1B for illustration of cue and probe period). Sixteen regions within the cognitive control network were identified that fit this cross-over pattern, including right inferior frontal junction (RIFJ), left inferior frontal gyrus (LIFG), and posterior parietal cortex (PPC) (see Figure 1A and Table 4). Event-related time courses were constructed to verify that patients showed reduced cue-related but increased probe- related activity (i.e. an increase in cue-related activity with a corresponding decrease in probe-related activity following training) (see Figure 1B).

To further examine group differences, we focused on cue-period activity and the relationship between A-cue and B-cue trials in the 16 ROIs identified in the above analysis. Previous studies have found that controls tend to show a stronger cue-related response on B-cue trials compared to A-cues while in patients these effects are weak or absent (Perlstein et al., 2003; Holmes et al., 2005; MacDonald et al., 2005; Yoon et al., 2008). A similar pattern was found in the current data (as reflected in a cue-type by group interaction) in several regions, including bilateral inferior frontal junction (see Table 4). Figure 2A illustrates this pattern in a representative region, the RIFJ $(x=+45$, $y=+10, z=+30)$. Prior to training, controls showed significantly greater B-cue activity than A-cue $(t(14)=5.02, p<0.001)$, but in patients, cue-period activation was more generally decreased, and there was no statistical difference in activation magnitude between the two cue types prior to training $(t(21)=0.88, p>0.2)$.

Next, we performed correlation analyses to examine the relationship between pre-training B-cue activation and patient symptoms. As expected, based on prior literature (Barch et al., 1999; Cohen et al., 1999), patients with more disorganized symptoms showed less B-cue activation in a number of the regions identified in the pre-training group difference analysis, including anterior cingulate cortex/presupplementary motor area, parietal cortex and medial frontal gyrus (ACC/pre-SMA) (PC: $r=-0.48, p<0.05$; ACC/preSMA: $r=-0.51, p<0.05)$. In addition, patients with more negative symptoms showed reduced activity in left IFJ $(r=-0.55, p<0.01)$. Further, we averaged activation across all 16 ROIs showing the crossover pattern of activation dynamics, and found that using this average
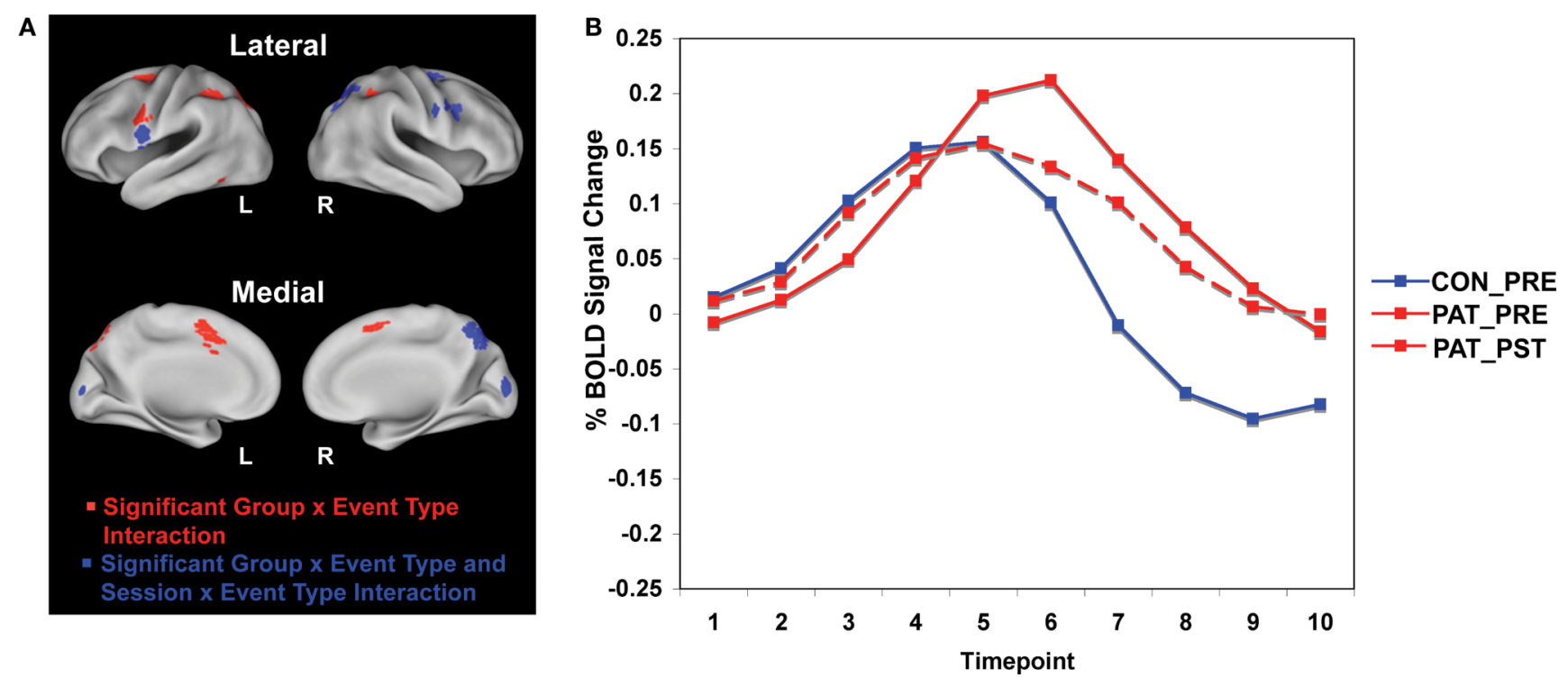

FIGURE 1 | (A) Brain regions showing group $\times$ event type interactions in patients and healthy controls and session $\times$ event type interactions in patients. Regions in red reflect greater blood oxygen level-dependent (BOLD) response at cue in controls relative to patients prior to training, and regions in blue reflect both greater BOLD response at cue in controls relative to patients prior to training and greater patient BOLD response at cue post-training relative to pretraining. (B) The time course plot shows control and patient pre-training and patient post-training cue and probe activity averaged across the nine regions showing both a group $\times$ event type interaction and a session $\times$ event type interaction shown in the images of the brain. 
Table 4 | Regions showing group differences and training effects in cue and probe-related activation.

\begin{tabular}{|c|c|c|c|c|c|}
\hline Region & $\begin{array}{l}\text { Brodmann } \\
\text { area }\end{array}$ & $X^{a}$ & $Y^{a}$ & $Z^{\mathrm{a}}$ & $\begin{array}{l}\text { Volume } \\
\left(\mathrm{mm}^{3}\right)^{\mathrm{b}}\end{array}$ \\
\hline Cerebellum & & -28 & -68 & -47 & 2916 \\
\hline Cerebellum & & +4 & -66 & -25 & 1377 \\
\hline Left temporal cortex & 37 & -45 & -54 & -11 & 270 \\
\hline L inferior frontal & 6 & -55 & +1 & +14 & 1377 \\
\hline R visual cortex & 17 & +8 & -88 & +7 & 864 \\
\hline L inferior frontal junction* & 9 & -51 & +3 & +28 & 891 \\
\hline$R$ inferior frontal junction ${ }^{*}$ & 9 & +45 & +10 & +30 & 405 \\
\hline R inferior frontal junction ${ }^{*} *$ & 6 & +37 & +0 & +25 & 216 \\
\hline R inferior frontal junction* & 6 & +52 & +2 & +32 & 540 \\
\hline Anterior cingulate & 32 & +0 & +7 & +46 & 2835 \\
\hline $\mathrm{R}$ inferior parietal cortex & 40 & +37 & -41 & +39 & 1161 \\
\hline L inferior parietal cortex & 40 & -34 & -44 & +42 & 1458 \\
\hline R superior parietal cortex & 7 & +19 & -64 & +47 & 5292 \\
\hline L precuneus & 7 & -12 & -69 & +45 & 1863 \\
\hline R middle frontal & 6 & +25 & -3 & +54 & 1890 \\
\hline L middle frontal & 6 & -22 & -2 & +52 & 1782 \\
\hline
\end{tabular}

${ }^{a} X, Y$, and $Z$ are coordinates in a standard stereotactic space (Talairach and Tournoux, 1988) in which positive values refer to regions right of $(X)$, anterior to $(Y)$, and superior to (Z) the anterior commisure.

${ }^{b}$ Volume refers to the number of voxels (converted to $\mathrm{mm}^{3}$ ) that reached statistical significance in each region of interest.

Bolded regions show a significant training effect li.e. session (pre-training/posttraining) by event type (cue/probe) interaction].

*Indicate regions in which healthy controls show significantly greater B-cue than A-cue activation prior to training while patients do not.

**Indicate regions that show a significant training effect and in which, following training, patients show significantly greater B-cue than A-cue activation whereas, prior to training, there was no significant difference between the cue types.

measure, disorganized symptoms were again correlated with less Bcue activation in the pre-training session $(r=-0.48, p<0.05)$ (see Figure 2B and Table 4). In contrast, disorganization was not significantly correlated with the magnitude of A-cue activity $(r=-0.27$, $p=0.23$ ), indicating that the effect was selective to B-cue activity. Positive symptoms were not correlated with activity in any region.

In summary, at baseline, controls showed greater cue-related activity than patients in a network of brain regions that are components of the canonical cognitive control network. In contrast, patients showed greater probe-related activity in controls in these same regions, which suggests that patients were relying on a more "reactive" cognitive control mode. In addition, activity was greater for B-cues than A-cues in bilateral frontal regions among controls, but not patients. Further, among patients, more severe disorganization symptoms were associated with less cue-related activity in a number of these cognitive control regions (e.g., RIFJ), especially for B-cue trials.

\section{Training Effects}

We examined whether training would produce a more proactive profile of increased cue-related but decreased probe-related activity. We again tested for a cross-over pattern of effects, but this time as a function of training session (i.e., session $\times$ event type interaction; cue: post > pre; probe: post < pre). To provide a strong test, this second stage was conducted as an ROI-based analysis on the 16 cognitive control regions identified as showing between-group differences related to an impairment in proactive control (see Figure 1A and Table 4).

A subset of nine of the original 16 regions were identified as showing training effects (see bolded regions in Table 4), reflected by a significant cross-over interaction in activity dynamics, including RIFJ, LIFG, and PPC. Event-related time courses verified that patients did indeed show a significant shift in activation from reactive to proactive control (i.e. shift showing increased cue-related activity and decreased probe-related activity - see Figure 1B). We further examined the effect of training broken down by cue type (B vs. A). The general pattern observed in the nine regions showing a significant session $\mathrm{X}$ cue/probe interaction was that the training increased B-cue activity to a greater extent than A-cue activity, and that B-cue $>$ A-cue effects were larger post training. This was statistically significant in two of the RIFJ regions, which demonstrated significant B $>$ A cue effects - similar to that observed in controls - after training, but not before (see Table 4). Figure 2A shows this effect in one of these RIFJ regions $(x=+45, y=+10$, $z=+30)$.

Finally, we examined whether the training-related shift in activation dynamics was associated with improvement in proactive control and/or symptoms. An activation shift score [(cue-probe)posttraining-(cue-probe)pre-training] was calculated for each patient, with higher activation shift scores indicating larger proactive shifts in activation (i.e. an increase in cue-related activity and decrease in probe-related activity). We correlated activation shift scores with task performance using the proactive control indices and found that a more proactive shift in activation in the RIFJ $(x=+52, y=+2$, $z=+32$ ) was associated with more improvement in proactive control in terms of the reaction time index $(r=-0.54, p=0.01)$. We also found, when restricting analyses to only the post-training session, that more cue-related activation in this same RIFJ region $(x=+52$, $y=+2, z=+32)$ was associated with greater proactive control in terms of RT ( $r=-0.47, p<0.05)$. Because we had previously found that B-cue activation was associated with disorganized and negative symptoms in the pre-training session, we examined whether training effects on B-cue activation were also associated with the severity of these symptoms. Higher disorganization scores were correlated with a larger training-related increase in B-cue activity in the same RIFJ region $(r=0.43, p<0.05)$. In addition, two other right IFJ regions were both positively correlated with negative symptoms scores $(+45 \pm 10 \pm 30: r=0.43, p<0.05 ;+37 \pm 0 \pm 25$ : $r=0.44, p<0.05$ ). Thus, patients with more disorganized and negative symptoms showed a greater benefit of training in terms of a larger increase in B-cue related activity (see Figure 3).

\section{DISCUSSION}

In the current study, we investigated whether an experimental manipulation designed to improve the use of cognitive control strategies in patients with schizophrenia would impact the dynamics of brain activity during task performance. The key findings were that this manipulation led to a significant shift in event-related activity within lateral PFC and related components of the brain cognitive control network, such that a more "proactive" pattern was 


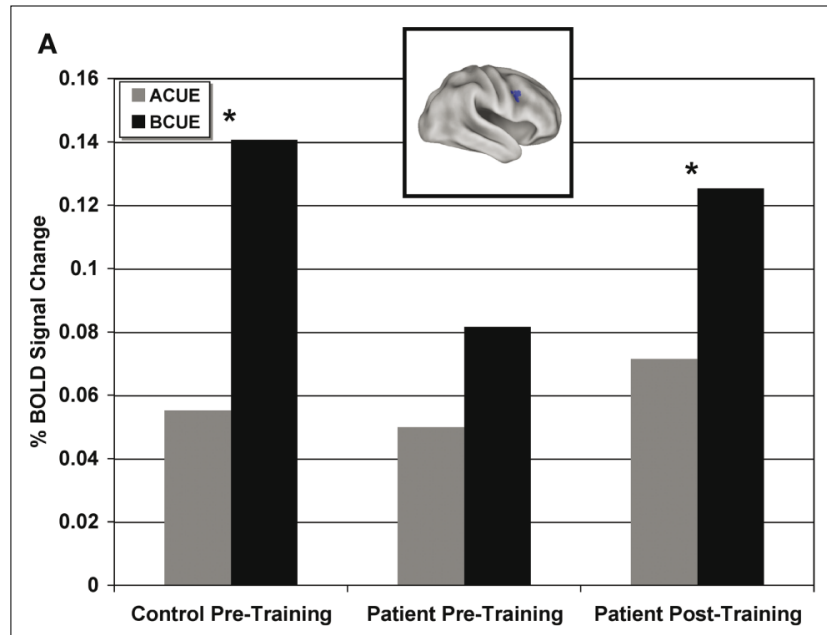

FIGURE 2 | (A) Right inferior frontal junction region $(x=+45, y=+10, z=+30)$ showing significantly greater $B$-cue relative to $A$-cue in healthy controls prior to training and significantly greater $B$-cue relative to $A$-cue in patients post-training. (B) Pre-training B-cue activity averaged across

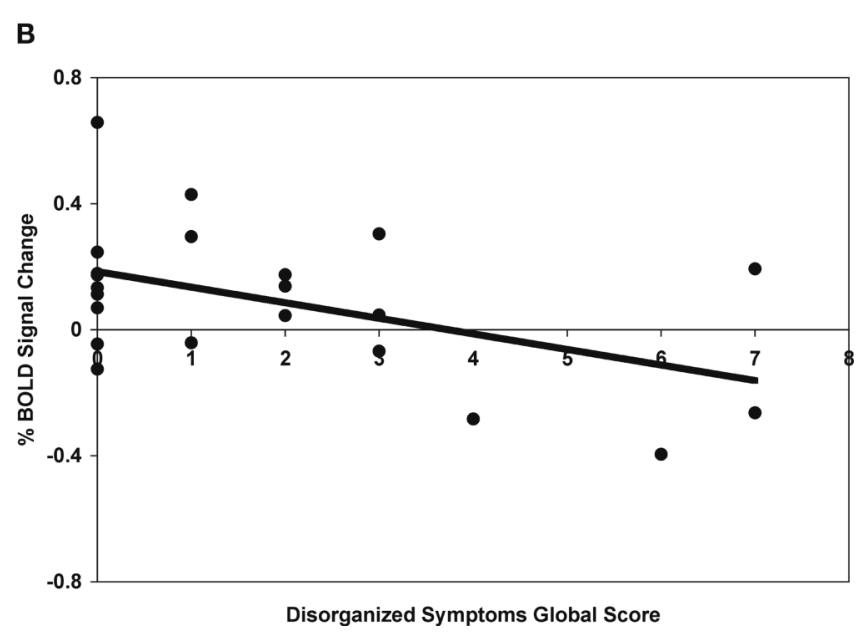

sixteen regions showing significant group by event type interactions negatively correlated with disorganized symptoms global score (includes disorientation from attention, positive formal thought disorder, and bizarre behavior).

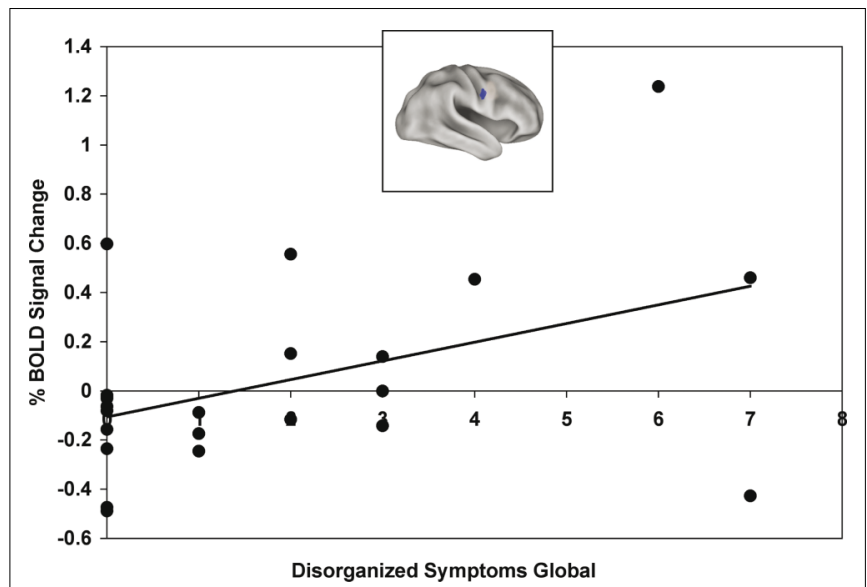

FIGURE 3 | Training-related improvement in B-Cue activation in right inferior frontal junction $(x=+52, y=+2, z=+32)$ positively correlated with disorganized symptoms global score (includes disorientation from attention, positive formal thought disorder, and bizarre behavior).

observed post-training, similar to that found in controls. Specifically, at baseline, patients with schizophrenia showed an activity pattern characteristic of a reactive control strategy, with reduced cue-related activation but increased activity during the probe period. After strategy training, cue-related activity increased while probe-related activity decreased, normalizing the post-training activity dynamics towards the control pattern. Moreover, these activity patterns appeared to be functionally important, as they were related to both clinical symptomatology (disorganization and negative symptoms) and training-related performance improvements associated with cognitive control (i.e., on BX trials). The results have a number of implications for our understanding of cognitive control in schizophrenia and its relationship to brain function.

\section{THE TEMPORAL DYNAMICS OF BRAIN ACTIVATION IN SCHIZOPHRENIA}

A critical take-home message from this study is that understanding the nature of functional brain changes in schizophrenia may necessitate a direct examination of the temporal dynamics of brain activity. The prior neuroimaging literature in schizophrenia has been subject to controversy regarding whether patients show hyper- or hypo-activation within brain regions associated with cognitive control (e.g., lateral PFC) (Callicott et al., 2000; Manoach, 2003; Van Snellenberg et al., 2006; Karlsgodt et al., 2009). One explanation put forward to explain this variability observed across studies is that hyper- vs. hypo-activation may be tightly linked with the respective behavioral performance levels exhibited in patient and control groups (Callicott et al., 2000; Van Snellenberg et al., 2006; Karlsgodt et al., 2009). Another explanation, derived from a recent meta-analysis by Minzenberg et al. (2009), is that different regions of PFC show hypo versus hyperactivation, with regions showing increased activity potentially reflecting compensatory processes. However, our results suggest yet another possible explanation. It may be the case that patients show reduced activation in some task components but increased activity in other components, which may not be resolved unless the experimental design and analysis explicitly permits examination of this issue. Thus, in the current study, by examining activity both during cue and probe periods within AX-CPT task trials, we found that patients showed opposite patterns of activity than controls in both periods. Relatedly, in other work, we have found that decomposition of temporal activity dynamics into sustained and transient components has also provided new insights into the nature of brain function differences between control groups and individuals at risk for schizophrenia (Brahmbatt and Barch, In submission). Our findings provide a possible resolution to the mixed results in the literature, by indicating how task paradigms or analysis approaches that blur over various task events (e.g., cue vs. probe) or components (e.g., sustained vs. transient, 
as in blocked designs) may not be able to resolve some of the complexities in activity dynamic changes that may be present in schizophrenia. Instead, the results may be biased in one direction or another based on specifics of the task design, and as such, may lead investigators to selectively interpret the results as supporting hypo or hyper-activation, as the case may be.

Another important aspect of the current findings is that the pattern of results permits stronger control against alternative interpretations. In particular, a major concern in neuroimaging studies comparing control and clinical populations is that the observed changes may be due to methodological factors, such as changes in brain structure, hemodynamics, signal-to-noise ratios, movement, or other confounds that may be present in the clinical group of interest. In the current results, the observed cross-over interaction in temporal activation dynamics between patients and controls, and the further cross-over shift in this activity within patients following training, would be hard to explain by any of these artifactual sources of group difference. Thus, we are relatively confident in interpreting results as a shift in activation dynamics among patients, rather than a simple increase or decrease in the magnitude of activity. Moreover, this shift pattern is very consistent with the hypotheses suggested by the DMC framework, that patients may tend to employ, by default, a reactive mode of cognitive control (marked by low cue-related activation, and thus a compensatory need to increase activity during probe periods), but that they may be able to shift into a more proactive mode, similar to controls, with sufficient training in optimal AX-CPT task strategies.

\section{CHANGES IN BRAIN ACTIVITY AS A FUNCTION OF TRAINING IN SCHIZOPHRENIA}

The fact that patients did show a shift in activation dynamics within the same cognitive control regions showing group differences at baseline supports ideas regarding the potential for changes in brain activity in response to strategy training. As such, this work makes contact with the recent literature focusing on the role of neuroplasticity in schizophrenia, which has prompted both cognitive rehabilitation projects as well as studies that have evaluated these efforts using brain monitoring techniques (Wykes et al., 2002b, 2007; Fisher et al., 2009a; Vinogradov et al., 2009). Our findings provide strong support for the idea that functional brain activity in individuals with schizophrenia can be altered by behavioral experiences, and moreover, that behaviorally-observed enhancements in cognition are accompanied by well-defined and interpretable changes in brain activation. This type of finding provides encouraging evidence that future studies of this type may show promise within schizophrenia, and should be undertaken with greater frequency. Further, our results also suggest a relationship between clinical symptom severity and effects of training. Individuals with greater severity of disorganization and negative symptoms at baseline showed the most improvement in both behavioral and brain activity indices of cognitive control. This finding is consistent with prior work by Uhlhaas et al. (2005), who found that spatial context processing was improved following treatment, but only in disorganized patients. Further, the degree of improvement in spatial context processing correlated with the degree of improvement in disorganization symptoms.
Nevertheless, our results also point to some of the limitations and challenges facing this work. First, although the strategy training in the AX-CPT led to clear and theoretically-interpretable changes in behavioral performance, there were still large differences in the performance patterns observed between patients and controls following training. This indicated significant residual AX-CPT impairments in the individuals with schizophrenia. It is possible that further performance gains may have been obtained with additional training, since our protocol was actually quite limited (a single session of about 30-45 min in duration). Nevertheless, the brain activation changes were quite extensive, showing a strong degree of normalization in dynamics relative to the control pattern. Indeed, the neural changes may have been stronger than observed behavioral changes. Thus, the functionality of regions that did not show training related changes may also have contributed to AX-CPT behavioral performance deficits in patients with schizophrenia.

A final point related to changes in brain activity as a function of intervention in schizophrenia is that it is still unknown whether changes in behavior and brain activity due to cognitive training reflect general cognitive enhancements, or a more narrow effect limited to the particular trained experiences. Specifically, the results of the current study provide no information regarding whether the AX-CPT effects would be maintained over longer intervals than an immediate post-training session, and more importantly, whether the training would positively impact brain activation and performance on other cognitive control tasks. Indeed, the issues of maintenance and transfer can be considered to be the "holy grails" in cognitive rehabilitation research, and are notoriously difficult to obtain (Wykes and Huddy, 2009). Thus, further studies will be needed to determine the extent to which the current results generalize beyond the single-session single-task patterns we observed.

\section{THE ROLE OF THE LATERAL PFC IN COGNITIVE CONTROL}

It is worth noting that the results we obtained from training in patients with schizophrenia are highly consistent with those that we observed in a prior study with healthy older adults (Braver et al., 2009). In both studies we found a very similar set of brain regions associated with cognitive control, centered on the lateral PFC, that showed cross-over shifts in brain activation dynamics as a function of AX-CPT strategy training. Although healthy older adults and patients with schizophrenia are very different in terms of behavioral and clinical profiles, there are some similarities in terms of impaired cognitive control functions. Thus, the results provide stronger support for the DMC framework in suggesting that similar functional patterns of reactive and proactive control can be found in diverse groups that show cognitive control impairments. As such, it may be the case that a shift from proactive to reactive control may serve as something like a final common pathway or mechanism that may result from a diverse set of etiological changes or pathologies.

Moreover, the common results from the two studies also point to specific brain regions that could serve as key functional loci underlying training-related enhancements in cognitive control. In both older adults and patients with schizophrenia, the RIFJ showed a classic reactive pattern at baseline, but a proactive shift following training. More importantly, in this region the activity dynamics and training-related changes were also associated with performance effects. In individuals with schizophrenia, increased cue-related 
activity in the RIFJ was associated with better performance on BX trials and reduced disorganization symptoms. Similarly, in older adults, training-related effects in RIFJ were associated with AX-CPT performance improvements associated with improved proactive control. Together, these results suggest that the RIFJ may serve a critical function in implementing the flexible use and updating of contextual cue information, a theoretical perspective that is consistent with other recent accounts of the functional role of this brain region (Brass et al., 2005).

\section{LIMITATIONS}

There are also several limitations to this study. As noted above, we do not know whether the training related changes we found would be maintained over time or generalize to other cognitive control tasks. It would be interesting to examine sleep-consolidation effects on cognitive control processes with a more extensive version of our cognitive control strategy training given over a 24 -h period, allowing us to examine the difference between a Day 1 and Day 2 session (as seen in Manoach et al., 2004). It is reasonable to hypothesize that sleep-related consolidation effects would indeed serve to increase the gains in cognitive control seen in patients. After exploring whether further effects in cognitive control can be gained through a more extensive version of our training protocol, the next step would be to see if the protocol can also show effects with other cognitive control and context processing tasks. In addition, our sample of individuals with schizophrenia were all taking medications, and it is difficult to know if we would have found the same results in unmedicated patients. However, as noted above, it would be hard to attribute our results to medication related confounds, as we saw double dissociations in both the group differences and in the training related effects. A more important limitation is that we did not retest the controls at the same interval as patients, and we did not include a no-intervention comparison group of patients. As such, it is theoretically possible that the changes we saw in patients reflect simple practice related changes. However, in prior work, we have

\section{REFERENCES}

Andreasen, N. C. (1983a). The Scale for the Assessment of Negative Symptoms (SANS). Iowa City, IA: University of Iowa.

Andreasen, N. C. (1983b). The Scale for the Assessment of Positive Symptoms (SAPS). Iowa City, IA: University of Iowa.

Andreasen, N.C. (1985). Positive vs. negative schizophrenia: a critical evaluation. Schizophr. Bull. 11, 380-389.

Andreasen, N. C., and Olsen, S. (1982). Negative vs. positive schizophrenia. Arch. Gen. Psychiatry 39, 789-794.

Barch, D. M., and Braver, T. S. (2005). Cognitive control in schizophrenia: cognitive and neural mechanisms, in Cognitive Limitations in Aging and Psychopathology: attention, Working Memory, and Executive Functions, eds R. W. Engle, Sedek, G., von Hecker, U., and McIntosh, D.N.

examined whether AX-CPT performance changes in individuals with schizophrenia over repeated assessments, and have not found evidence for the kind of theoretically interpretable changes that we saw in the current study (Barch et al., 2003). Thus, while future studies will need to include additional control groups, we do think the current results provide encouraging support for the utility of cognitive training approaches in modulating behavior and brain activity in schizophrenia. Further, it will be important in future work to determine whether the enhancements found in AX-CPT task performance as a result of proactive strategy training translate to real world contexts in which the use of such proactive control strategies could - at least in theory - improve life function.

\section{CONCLUSION}

In summary, the current study contributes to the literature on the neural basis of cognitive control impairments in schizophrenia, by demonstrating that these relate to a complex shift in the temporal dynamics of brain activity, which may reflect the adoption of a reactive control strategy. More importantly, the observation that these brain activation dynamics can be significantly impacted by even a short theoretically motivated strategy training session indicates the potential for modification of cognitive control functions in schizophrenia. Our theoretical framework of the DMC model provides one perspective on how such plasticity might occur - via training-related shifts from a more reactive form of cognitive control to a more proactive form, normalizing to the pattern postulated to be typically adopted by healthy controls. The extent to which the DMC model provides a good account of cognitive control changes in schizophrenia still remains to be tested through follow-up and theoretically directed studies.

\section{ACKNOWLEDGMENTS}

This research was funded by a National Alliance for Research on Schizophrenia (NARSAD) Independent Investigator award to Todd S. Braver. We would like to thank the individuals with schizophrenia who participated in this study.

of distractibility: the mechanisms underlying increased Stroop effects in schizophrenia. Schizophr. Bull. 24, 749-762.

Brass, M., Derrfuss, J., Forstmann, B., and von Cramon, D. Y. (2005). The role of the inferior frontal junction area in cognitive control. Trends Cogn. Sci. (Regul. Ed.) 9, 314-316.

Braver, T. S., Barch, D. M., and Cohen, J. D. (1999). Cognition and control in schizophrenia: a computational model of dopamine and prefrontal function. Biol. Psychiatry 46, 312-328.

Braver, T. S., and Cohen, J. D. (2000). "On the control of control: the role of dopamine in regulating prefrontal function and working memory," in Attention and Performance XVIII. eds S. Monsell and J. Driver (Cambridge, MA: MIT Press), 713-738.

Braver, T.S., Cohen, J. D., and Barch, D. M. (2002). "The role of the prefrontal cor- tex in normal and disordered cognitive control: a cognitive neuroscience perspective," in Principles of Frontal Lobe Function. eds D. T. Stuss and R. T. Knight (Oxford: Oxford University Press), 428-448.

Braver, T. S., Gray, J. R., and Burgess, G. C. (2007). Explaining the many varieties of working memory variation: dual mechanisms of cognitive control, in Variation in Working memory, eds A. Conway, M. J. Kane, A. Miyake, and J. Towse, (Oxford: Oxford University Press), 76-106.

Braver, T. S., Paxton, J. L., Locke, H. S., and Barch, D. M. (2009). Flexible neural mechanisms of cognitive control within human prefrontal cortex. Proc. Natl. Acad. Sci. U.S.A. 106, 7351-7356.

4-week course, and relationships to clinical symptoms. J. Abnorm. Psychol. 112, 132-143.

Barch, D. M., Carter, C. S., Hachten, P. C., and Cohen, J.D. (1999). The "benefits"
Callicott, J.H., Bertolino, A., Mattay, V. S., Langheim, F. J., Duyn, J., Coppola, R., Goldberg, T. E., and Weinberger, D. R. (2000). Physiological dysfunction of 
the dorsolateral prefrontal cortex in schizophrenia revisited. Cereb. Cortex. 10, 1078-1092.

Cohen, J. D., Barch, D. M., Carter, C., and Servan-Schreiber,D. (1999a). Contextprocessing deficits in schizophrenia: converging evidence from three theoretically motivated cognitive tasks. $J$. Abnorm. Psychol. 108, 120-133.

Cohen, J. D., Barch, D. M., Carter, C. S., and Servan-Schreiber, D. (1999b). Schizophrenic deficits in the processing of context: converging evidence from three theoretically motivated cognitive tasks. J. Abnorm. Psychol. 108, 120-133.

Cohen, J. D., Braver, T. S., and O'Reilly, R. (1996). A computational approach to prefrontal cortex, cognitive control, and schizophrenia: recent developments and current challenges. Philos. Trans. R. Soc. Lond., B, Biol. Sci. 351, 1515-1527.

Cohen, J. D., MacWhinney, B., Flatt, M., and Provost, J. (1993). PsyScope: a new graphic interactive environment for designing psychology experiments. Behav. Res. Methods Instrum Comput. 25, 257-271.

Cohen, J. D., and Servan-Schreiber, D. (1992).Context, cortex and dopamine: a connectionist approach to behavior and biology in schizophrenia. Psychol. Rev. 99, 45-77.

Conturo, T.E., McKinstry, R. C., Akbudak, E., Snyder, A. Z., Yang, T., and Raichle, M.E. (1996). Sensitivity optimization and experimental design in functional magnetic resonance imaging. Abstr. Soc. Neurosci. 26, 7.

Corrigan, P. W., Hirschbeck, J. N., and Wolfe, M. (1995). Memory and vigilance training to improve social perception in schizophrenia. Schizophr. Res. 17, 257-265.

Davidson, L. L., and Heinrichs, R. W. (2003). Quantification of frontal and temporal lobe brain-imaging findings in schizophrenia: a meta-analysis. Psychiatry Res.: Neuroimaging 122, 69-87.

Emery, L., Heaven, T. J., Paxton, J. L., and Braver, T. S. (2008). Age-related changes in neural activity during performance matched working memory manipulation. Neuroimage 42, 1577-1586.

Fales, C. L., Barch, D. M., Burgess, G. C., Schaefer, A., Mennin, D. S., Gray, J. R., and Braver, T. S. (2008). Anxiety and cognitive efficiency: differential modulation of transient and sustained neural activity during a working memory task. Cogn. Affect. Behav. Neurosci. 8, 239-253.

First, M. B., Spitzer, R. L., Gibbon, M., and Williams, J. B. W. (2001). Structured Clinical Interveiw for DSM-IV-TR Axis Disorders. New York: Biometrics
Research Department, New York State Psychiatric Institute.

Fisher, M., Holland, C., Merzenich, M. M., and Vinogradov, S. (2009a). Using neuroplasticity-based auditory training to improve verbal memory in schizophrenia. Am. J. Psychiatry 166, 805-811.

Fisher, M., Holland, C., Subramaniam, K., and Vinogradov, S. (2009b). Neuroplasticity-based cognitive training in schizophrenia: an interim report on the effects 6 months later.Schizophr. Bull. Epub ahead of print.

Friston, K. J., Holmes, A. P., Worsley, K. J., Poline, J. P., Frith, C. D., and Frackowiak, R. S. J. (1995). Statistical parametric mapping and other analyses of functional imaging data. Hum. Brain Mapp. 2, 189-210.

Friston, K. J., Williams, S., Howard, R., Frackowiak, R. S. J., and Turner, R. (1996). Movement-related effects in fMRI time-series. Magn. Reson. Med. 35, 346-355.

Glahn, D. C., Ragland, J. D., Abramoff, A., Barrett, J., Laird, A. R., Bearden, C. E., and Velligan, D.I. (2005). Beyond hypofrontality: A quantitative meta-analysis of functional neuroimaging studies of working memory in schizophrenia. Hum. Brain Mapp. 25, 60-69.

Goldman-Rakic, P. S. (1991). "Prefrontal cortical dysfunction in schizophrenia: the relevance of working memory," in Psychopathology and the Brain, eds B. J. Carroll and J. E. Barrett (New York: Raven Press, Ltd.), 1-23.

Goldman-Rakic, P. S., and Selemon, L. D. (1997). Functional and anatomical aspects of prefrontal pathology in schizophrenia. Schizophr. Bull. 23, 437-458.

Haut, K. M., Lim, K. O. and MacDonald, A., (in press). Prefrontal cortical changes following cognitive training in patients with schizophrenia: effects of practice, generalization, and specificity. Neuropsychopharmacology.

Holmes, A. J., MacDonald, A., Carter, C. S., Barch, D. M., Stenger, V. A., and Cohen, J. D. (2005). Prefrontal functioning during context processing in schizophrenia. Psychiatry Res 110, 137-149.

Javitt, D. C., Shelley, A., Silipo, G., and Lieberman, J. A. (2000). Deficits in auditory and visual context-dependent processing in schizophrenia. Arch. Gen. Psychiatry 57, 1131-1137.

Karlsgodt, K. H., Sanz, J., van Erp, T. G., Bearden, C. E., Nuechterlein, K. H., and Cannon, T. D. (2009). Re-evaluating dorsolateral prefrontal cortex activation during working memory in schizophrenia. Schizophr. Res. 108, 143-150.

Lewine, R. J., Fogg, L., and Meltzer, H. Y. (1983). Assessment of negative and positive symptoms in schizophrenia. Schizophr. Bull. 9, 368-376.

Liddle, P. F. (1987). Syndromes of chronic schizophrenia: a re-examination of the positive-negative dichotomy. $\mathrm{Br}$. J. Psychiatry 151, 145-151.

MacDonald, A. W. III, and Carter, C. S. (2003). Event-related FMRI study of context processing in dorsolateral prefrontal cortex of patients with schizophrenia. J. Abnorm. Psychol. 112, 689-697.

MacDonald, A. W. III, Cohen, J. D., Stenger, V.A., and Carter, C. S. (2000). Dissociating the role of the dorsolateral prefrontal and anterior cingulate cortex in cognitive control. Science 288, 1835-1838.

MacDonald, A. W., Carter, C. S., Kerns, J. G., Ursu, S., Barch, D. M., Holmes, A. J., Stenger, V.A., and Cohen, J. D. (2005). Specificity of prefrontal dysfunction and context processing deficits in schizophrenia in never-medicated patients with first-episode psychosis. Am. J. Psychiatry 162, 475-484.

MacDonald, A. W., Pogue-Geile, M. F., Johnson, M. K., and Carter, C. S. (2003). A specific deficit in context processing in the unaffected siblings of patients with schizophrenia. Arch. Gen. Psychiatry 60, 57-65.

Manoach, D. S. (2003). Prefrontal cortex dysfunction during working memory performance in schizophrenia: reconciling discrepant findings. Schizophr. Res. 60, 285-298.

Manoach, D.S., Cain, M. S., Vangel, M. G., Khurana,A., Goff, D.C., and Stickgold, R. (2004). A failure of sleep-dependent procedural learning in chronic, medicated schizophrenia. Biol. Psychiatry 56, 951-956.

Miller, D. D., Arndt, S., and Andreasen, N. C. (1993). Alogia, attentional impairment, and inappropriate affect: their status in the dimensions of schizophrenia. Compr. Psychiatry 34, 221-226.

Minzenberg, M. J., Laird, A. R., Thelen, S., Carter, C. S., and Glahn, D. C. (2009). Meta-analysis of 41 functional neuroimaging studies of executive function in schizophrenia. Arch. Gen. Psychiatry 66, 811-822.

Oldfield, R. C. (1971). The assessment and analysis of handedness: the Edinburgh inventory. Neuropsychologia 9, 97-113.

O’Reilly, R. C., Braver, T. S., and Cohen, J. D. (1999). "A biologically-based computational model of working memory," in Models of Working Memory: Mechanisms of Active Maintenance and Executive Control, eds A. Miyake and P. Shah, (New York: Cambridge University Press), 375-411.

Owen, A. M., McMillan, K. M., Laird, A. R., and Bullmore, E. (2005). N-Back working memory paradigm: a meta- analysis of normative functional neuroimaging studies. Hum. Brain Mapping 25, 46-59.

Paxton, J. L., Barch, D. M., Racine, C. A., and Braver, T.S. (2008). Cognitive control, goal maintenance, and prefrontal function in healthy aging. Cereb. Cortex 18, 1010-1028.

Paxton, J. L., Barch, D. M., Storandt, M., and Braver, T. S. (2006). Effects of environmental support and strategy training on older adults' use of context. Psychol. Aging 21, 499-509.

Perlstein, W. M., Dixit, N. K., Carter, C. S., Noll, D. C., and Cohen, J. D. (2003). Prefrontal cortex dysfunction mediates deficits in working memory and prepotent responding in schizophrenia. Biol. Psychiatry 53, 25-38.

Reichenberg, A., and Harvey, P.D. (2007). Neuropsychological impairments in schizophrenia: integration of performance-based and brain imaging findings. Psychol. Bull. 133, 833-858.

Servan-Schreiber, D., Cohen, J. D., and Steingard, S. (1996). Schizophrenic deficits in the processing of context: a test of a theoretical model. Arch. Gen. Psychiatry 53, 1105-1113.

Snyder, A. Z. (1996). "Difference image versus ratio image error function forms in PET-PET realignment," in Quantification of Brain Function Using PET, eds D. Bailer, and T. Jones (San Diego: Academic Press), pp. 131-137.

Talairach, J., and Tournoux, P. (1988). Coplanar stereotaxic atlas of the human brain. Thieme, New York.

Toomey, R., Kremen, W. S., Simpson, J. C., Samson, J. A., Seidman, L. J., Lyons, M. J., Faraone, S. V., and Tsuang, M. T. (1997). Revisiting a factor structure for positive and negative symptoms: evidence from a large heterogeneous group of psychiatric patients. Am. J. Psychiatry 154, 371-377.

Twamley, E. W., Jeste, D. V., and Bellack, A. S. (2003). A review of cognitive training in schizophrenia. Schizophr. Bull. 29, 359-382.

Twamley, E. W., Savla, G. N., Zurhellen, C. H., Heaton, R. K., and Jeste, D. V. (2008). Development and pilot testing of a novel compensatory cognitive training intervention for people with psychosis. Am. J. Psychiatr. Rehabil. 11, 144-163.

Uhlhaas, P. J., Phillips, W. A., and Silverstein, S. M. (2005). The course and clinical correlates of dysfunctions in visual perceptual organization in schizophrenia during the remission of psychotic symptoms. Schizophr. Res. 75, 183-192.

Van Snellenberg, J. X., Torres, I. J., and Thornton, A. E. (2006). Functional neuroimaging of working memory in 
schizophrenia: task performance as a moderating variable. Neuropsychology 20, 497-510.

Vinogradov, S., Fisher, M., Warm, H., Holland, C., Kirshner, M. A., and Pollock, B. G. (2009). The cognitive cost of anticholinergic burden: decreased response to cognitive training in schizophrenia. Am. J. Psychiatry 166, 1055-1062.

Wager, T. D., and Smith, E. E. (2003). Neuroimaging studies of working memory: a meta-analysis. Cogn. Affect. Behav. Neurosci. 3, 255-274.

Wechsler, D. (1997). Wechsler Adult Intelligence Scale - 3rd Edn. San Antonio: The Psychological Corporation.

Woods, R.P., Cherry, S. R., and Mazziotta, J. C. (1992). Rapid automated algorithm for aligning and reslicing PET images. J. Comput. Assist. Tomogr. 16, 620-633.
Woods, R. P., Grafton, S. T., Holmes, C. J., Cherry, S. R., and Mazziotta, J. C. (1998). Automated image registration: I. general methods and intrasubject, intramodality validation. J. Comput. Assist. Tomogr. 22, 139-152.

Wykes, T., Brammer, M., Mellers, J., Bray, P., Reeder, C., Williams, C., and Corner, J. (2002a). Effects on the brain of a psychological treatment: cognitive remediation therapy. Br. J. Psychiatry 181, 144-152.

Wykes, T., Brammer, M., Mellers, J., Bray, P., Reeder, C., Williams, C., and Corner, J. (2002b). Effects on the brain of a psychological treatment: cognitive remediation therapy: functional magnetic resonance imaging in schizophrenia. Br. J. Psychiatry 181, 144-152.

Wykes, T., and Huddy, V. (2009). Cognitive remediation for schizophrenia: it is even more complicated. Curr. Opin. Psychiatry 22, 161-167.

Wykes, T., Reeder, C., Landau, S., Everitt, B., Knapp, M., Patel, A., and Romeo, R. (2007). Cognitive remediation therapy in schizophrenia: randomized controlled trial. Br. J. Psychiatry 190, 421-427.

Yoon, J. H., Minzenberg, M. J., Ursu, S., Walters, R., Wendelken, C., Ragland, J. D., and Carter, C. S. (2008). Association of dorsolateral prefrontal cortex dysfunction with disrupted coordinated brain activity in schizophrenia: Relationship with impaired cognition, behavioral disorganization, and global function. Am. J. Psychiatry 165, 1006-1014.

Conflict of Interest Statement: The authors declare that the research was conducted in the absence of any com- mercial or financial relationships that could be construed as a potential conflict of interest.

Received: 07 August 2009; paper pending published: 11 September 2009; accepted: 25 March 2010; published online: 26 April 2010.

Citation: Edwards BG, Barch DM and Braver TS (2010) Improving prefrontal cortex function in schizophrenia through focused training of cognitive control. Front. Hum. Neurosci. 4:32. doi: 10.3389/fnhum.2010.00032

Copyright (C) 2010 Edwards, Barch and Braver. This is an open-access article subject to an exclusive license agreement between the authors and the Frontiers Research Foundation, which permits unrestricted use, distribution, and reproduction in any medium, provided the original authors and source are credited. 